\title{
Seroepidemiological aspects of human infection by Strongyloides stercoralis in Alfenas, southern Minas Gerais, Brazil
}

\author{
Juliana Barbosa Nunes ${ }^{[1]}$, Thamy Cristina de Oliveira Emídio ${ }^{[1]}$, Marcos José Marques ${ }^{[1]}$, \\ Ivo Santana Caldas ${ }^{[1]}$, Raquel Lopes Martins Souza ${ }^{[1]}$, \\ Herminia Yohko Kanamura ${ }^{[1]}$ and Julia Maria Costa-Cruz ${ }^{[2]}$
}

\author{
[1]. Departamento de Patologia e Parasitologia, Instituto de Ciências Biomédicas, \\ Universidade Federal de Alfenas, Alfenas, MG, Brasil. \\ [2]. Laboratório de Diagnóstico de Parasitoses, Departamento de Parasitologia, Instituto de Ciências Biomédicas, \\ Universidade Federal de Uberlândia, Uberlândia, MG, Brasil.
}

\begin{abstract}
Introduction: In most Strongyloides stercoralis infected individuals, nematoidosis occurs asymptomatically, but in immunocompromised patients, it can cause hyperinfection. Serological techniques seem to be a good alternative for detecting this parasite. Methods: The frequency of seropositivity for strongyloidiasis in Alfenas, MG, was estimated using the enzyme linked immunosorbent assay on blood samples, between May and August of 2015. Results: Out of 258 samples tested, 53.9\% were positive, and the frequency of seropositive individuals was higher in the peripheral districts of the municipality. Conclusions: The results indicate high seropositivity rates for strongyloidiasis among the residents of Alfenas city.
\end{abstract}

Keywords: Strongyloidiasis. Helminth, Seropositivity. Alfenas.

Human strongyloidiasis is a helminth disease caused by Strongyloides stercoralis, infecting 30-100 million people worldwide ${ }^{1}$. This infection is more common in hot and humid regions, where sanitary conditions are unsatisfactory or non-existent and most infected people present a chronic asymptomatic infection. Due to this autoinfection cycle, individuals with $S$. stercoralis infection can remain infected lifelong under normal conditions, if not properly treated and are generally asymptomatic. However, immunosuppressed individuals, especially those on corticosteroids can develop a hyperinfection syndrome with symptoms of severe disease, which can be fatal2,3.

The prevalence of strongyloidiasis has increased, particularly in the southern, eastern and central Europe, Caribbean islands, Southeast Asia, Latin America, and sub-Saharan Africa ${ }^{4}$. In non-endemic regions, it is mainly diagnosed in migrants from endemic countries ${ }^{3}$. In many tropical and subtropical countries, the prevalence of S. stercoralis infection ranges from $10 \%$ to $40 \%{ }^{1}$, reaching $50 \%$ in areas where moist soil and inadequate

Corresponding author: Juliana Barbosa Nunes.

e-mail: juliana_bnunes@yahoo.com.br

Received 4 March 2018

Accepted 15 May 2018 disposal of human waste coexist, such as certain tropical regions of Brazil ${ }^{4}$.

Compared to other soil-transmitted helminths (STHs), $S$. stercoralis infection is difficult to diagnose. The slow release of larvae in the feces leads to a low parasite load in most infected individuals making parasitological methods less sensitive $e^{4,5}$. The infection is systematically missed by diagnostic methods recommended by the World Health Organization (WHO), such as the Kato-Katz method ${ }^{1}$. Besides, the parasitological methods are labor-intensive, and difficult to perform due to the presence of infective live larvae, especially in field studies 5 .

Several immunological tests, such as the enzymelinked immunosorbent assay (ELISA), western blot, immunofluorescence, and the luciferase immunoprecipitation system, have been described for the diagnosis of strongyloidiasis, and have been demonstrated to have better sensitivity than the parasitological techniques. ELISA has a sensitivity ranging from $57 \%$ to $100 \%$, and specificity from $60 \%$ to $100 \%$ depending on the characteristics of the serum sample and the antigenic preparation ${ }^{4}$. Although problems related to cross-reactivity with other nematodes must be considered, these immunological methods are promising as screening tools in clinical and epidemiological studies ${ }^{4,5}$.

The present study aimed to estimate the serological frequency of strongyloidiasis in the municipality of Alfenas, 
Minas Gerais, using ELISA on blood samples collected from patients who attended the Central Laboratory of Clinical Analyses (Laboratório Central de Análises Clínicas, LACEN) of the Federal University of Alfenas, UNIFAL-MG.

The study was carried out in the municipality of Alfenas, located in the southern region of the state of Minas Gerais, Brazil. Alfenas is located $343 \mathrm{~km}$ away from Belo Horizonte, the capital of the state, and was reported to have a population of 73,774 inhabitants in the most recent census (2010) and an estimated population of 79,707 inhabitants in $2017^{6}$.

A total of 258 serum samples were selected between May and August 2015. The samples diluted with an equal volume of buffered glycerin (neutral $\mathrm{pH}$ ) to improve preservation, were identified based on their LACEN database code and were stored at $-20^{\circ} \mathrm{C}$ until further use. Anonymized information regarding the gender, age and residence address of the patients was collected from the LACEN database and organized in a Microsoft Office Excel® (Microsoft Corporation, Redmond, WA, USA) worksheet for further analysis.

The ELISA was performed according to a previously described method ${ }^{7}$. Briefly, polystyrene microplates were coated with detergent extract of Strongyloides venezuelensis $(50 \mu \mathrm{L} /$ well) at a final concentration of $5 \mu \mathrm{g} / \mathrm{mL}$ in $0.06 \mathrm{M}$ carbonatebicarbonate buffer ( $\mathrm{pH} 9.6)$. After overnight incubation at $4{ }^{\circ} \mathrm{C}$, the plates were washed three times for 5 min with phosphatebuffered saline (PBS, pH 7.2) containing $0.05 \%$ Tween 20 (PBS-T), and then blocked with PBS-T plus $3 \%$ non-fat milk (PBS-TM) for $45 \mathrm{~min}$ at $37^{\circ} \mathrm{C}$. Serum samples were diluted 1:100 in PBS-TM and incubated for $45 \mathrm{~min}$ at $37^{\circ} \mathrm{C}$. After washing the plates, peroxidase-conjugated goat anti-human IgG antibodies (Sigma, St. Louis, MO, USA) diluted in PBS-TM at a previously determined dilution were added to each well and incubated for $45 \mathrm{~min}$ at $37^{\circ} \mathrm{C}$. The reaction was visualized by the addition of the enzyme substrate $\left(0.03 \% \mathrm{H}_{2} \mathrm{O}_{2}\right)$ and chromogen (ortho-phenylenediamine) in $0.1 \mathrm{M}$ phosphate-citrate buffer $(\mathrm{pH}$ 5.0). After incubation in the dark for $15 \mathrm{~min}$ at room temperature, the reaction was stopped by the addition of $2 \mathrm{~N} \mathrm{H}_{2} \mathrm{SO}_{4}$ solution. Optical densities (OD) were determined at $492 \mathrm{~nm}$ using an ELISA reader (Anthos-Labtec Zenyt 200rt, Cambridge, UK). Cutoff values were calculated each day using the arithmetic mean OD of three negative control samples, performed in duplicate, plus three standard deviations. The reactivity index (RI) was determined by calculating the relationship between the OD of the sample and the cutoff value for that day according to the formula: $\mathrm{RI}=\mathrm{OD} /$ cutoff. Values of $\mathrm{RI}>1$ were considered positive. The positivity rates were determined according to gender, age, and place of residence.

Data were analyzed using the Epi Info ${ }^{\mathrm{TM}}$ software (version 7.2.1.0; Centers for Disease Control and Prevention, Atlanta, GA, USA). The data were reported as percent seropositive (positivity rates), and the respective $95 \%$ confidence intervals were calculated to verify the significance of the differences observed among the diverse groups.

The strongyloidiasis seropositivity data were evaluated in the districts/neighborhoods of Alfenas which were organized into seven regions, as shown in Figure 1. Region A represented the central area of the city and region $\mathrm{G}$ included two rural areas, the Gaspar Lopes district and the Community of Barbaras.

Of the 258 serum samples tested, 139 (53.9\%) were positive for strongyloidiasis. The positivity rates in women and men were $52.4 \%$ and $56.7 \%$, respectively, with no significant difference between genders.

Table 1 shows the distribution of strongyloidiasis seropositivity in regions A to G of Alfenas (Figure 1). The frequency of seropositivity was higher in the neighborhoods/ districts at the periphery of the municipality $(E, F$, and $G)$ compared to those within the central region $(\mathrm{A}, \mathrm{B}, \mathrm{C}$, and D), though the differences were not significant. The highest positivity rates were observed in the age groups of 31 to 40 years $(62.5 \%)$ and 41 to 50 years (60\%) (Table 2). However, the differences between age groups were not significant (Table 2).

Soil-transmitted helminth (STH) infections continue to represent a major public health issue in many developing countries. STHs are transmitted via the eggs or larvae in the feces of infected individuals, which in turn contaminate the soil in areas with poor sanitation. It is estimated that approximately 1.5 billion people are infected with STHs worldwide, but this could be an underestimation due to a large number of undiagnosed individuals ${ }^{8}$. From a clinical-epidemiological standpoint, strongyloidiasis is relevant because if not treated properly, it can persist for life in various severe forms some of which can be fatal. Many epidemiological aspects of $S$. stercoralis infection are poorly understood, with all the available information coming from studies on other STHs which mostly use diagnostic methods with low-sensitivity for $S$. stercoralis $^{1}$. The Kato-Katz technique is based on the presence of helminth eggs, but cannot detect larvae, which is the basis for the diagnosis of strongyloidiasis. Some parasitological methods that are based on the parasite load such as spontaneous sedimentation or formalin-ether centrifugation, also have low sensitivity for detecting larvae. However, the Baermann technique and its variations, as well as agar plate culture can detect larvae, the latter being more sensitive. These have been shown to be more effective methods for the parasitological diagnosis of strongyloidiasis ${ }^{9}$. However, the low parasitic burden in most patients with chronic infections caused due to the intermittent larval excretion results in a high number of false negatives ${ }^{3}$.

Like in other countries, strongyloidiasis is considered a neglected disease in Brazil. Based on parasitological methods, its occurence in humans is $5.3 \%, 7.9 \%, 6.6 \%, 3.9 \%$ and $4 \%$ in the north, northeast, midwest, southeast and the south of Brazil ${ }^{10}$. Few serological surveys for $S$. stercoralis have been conducted in Brazil, which have focused on the state of Minas Gerais in southeast Brazil. Using an immunofluorescence antibody test (IFAT), the mean rate of positivity in different populations has been reported to range from $7.7 \%$ to $41.1 \%$, and from $18.5 \%$ to $40.0 \%$ as measured by ELISA-IgG ${ }^{11,12}$.

In the present study, which included patients attending a clinical laboratory in Alfenas, a positivity rate of $53.9 \%$ $(139 / 258)$ was observed using a detergent extract of $S$. venezuelensis as the antigen in the ELISA-IgG test. According to da Silva et al. ${ }^{7}$ this test has a sensitivity of $83 \%$ and a 


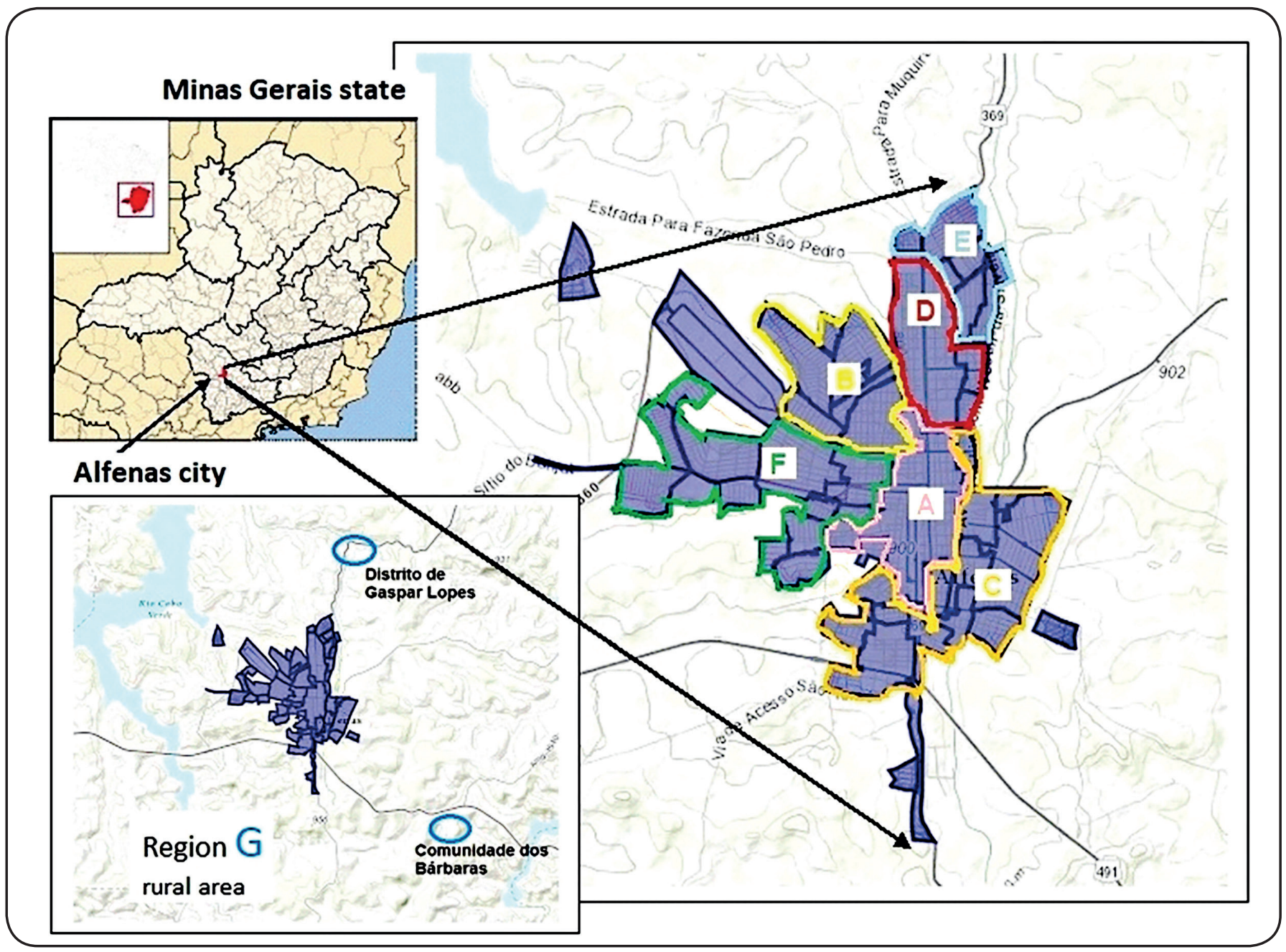

FIGURE 1: Localization of Alfenas city in the south region of Minas Gerais state, and organization of its different neighborhoods/districts as regions (A to G).

Fonte: <https://www.arcgis.com/home/webmap/viewer.html?webmap=5a0f0edb7c05451b8dc9b8dbc9e7e519> and <https://pt.wikipedia.org/wiki/Alfenas>, with some modifications introduced by the authors.

TABLE 1: The occurence of anti-Strongyloides seropositivity in different regions of Alfenas.

\begin{tabular}{cccc}
\hline Region & $\mathbf{N}$ & $\mathbf{P}$ & \% P (95\% confidence interval) \\
A & 24 & 22 & $47.8(32.9-63.1)$ \\
B & 22 & 23 & $51.1(35.8-66.3)$ \\
C & 10 & 4 & $28.6(8.4-58.1)$ \\
D & 23 & 23 & $50.0(34.9-65.1)$ \\
E & 24 & 37 & $60.7(47.3-72.9)$ \\
F & 9 & 21 & $70.0(50.6-85.3)$ \\
G & 2 & 7 & $77.8(40.0-97.2)$ \\
Without information & 5 & 2 & $28.6(3.7-71.0)$ \\
\hline Total & 119 & 139 & $\mathbf{5 3 . 9 ( 4 7 . 6 - 6 0 . 1 )}$ \\
\hline
\end{tabular}

Shown are the positivity rates (\% P) of anti-Strongyloides IgG antibodies, detected by ELISA in 258 patients living in Alfenas, MG, who attended the Central Laboratory of Clinical Analysis (LACEN) of the Federal University of Alfenas, from May to August 2015. The patients have been categorized based on the regions of their residence, within the municipality of Alfenas.

$\mathrm{N}$ (Negative) or $\mathrm{P}$ (Positive), if $\mathrm{RI} \leq 1.0$ and $\mathrm{RI}>1.0[\mathrm{RI}=$ reactivity Index = OD of the sample / Cut Off], respectively. 
TABLE 2: The occurence of anti-Strongyloides seropositivity in different age groups.

\begin{tabular}{|c|c|c|c|}
\hline Age group (years) & $\mathbf{N}$ & $\mathbf{P}$ & $\% \mathrm{P}(95 \%$ confidence interval) \\
\hline $21-30$ & 17 & 24 & $58.5(42.1-73.7 \%)$ \\
\hline $41-50$ & 14 & 21 & $60.0(42.1-76.1 \%)$ \\
\hline $51-60$ & 19 & 23 & $54.8(38.7-70.2 \%)$ \\
\hline $61-70$ & 18 & 12 & $40.0(22.7-59.4 \%)$ \\
\hline
\end{tabular}

Shown are the positivity rates (\% P) of anti-Strongyloides IgG antibodies, detected by ELISA, in the 258 patients living in Alfenas, MG, attending at the Central Laboratory of Clinical Analysis (LACEN) of the Federal University of Alfenas, from May to August 2015. The patients have been categorized based on their age.

$\mathrm{N}$ (Negative) or $\mathrm{P}$ (Positive), if $\mathrm{RI} \leq 1.0$ and $\mathrm{RI}>1.0$ [RI = reactivity Index = OD of the sample / Cut off], respectively.

specificity of $86 \%$. The seroprevalence for strongyloidiasis observed in this study area is higher than that in northwestern Minas Gerais, which reported prevalence rates of $18 \%$ to $40 \%$. Serological surveys conducted in other Latin American countries have shown contrasting results. In Peru, in a rural community of the Amazon region, a seroprevalence of $72 \%(442 / 609)$ was found by ELISA, whereas $S$. stercoralis was identified in only $8.7 \%(69 / 792)$ of the stool samples evaluated by three parasitological techniques including the Baermann method. ${ }^{13} \mathrm{In}$ Chile, $0.25 \%$ (3/1200) of the samples from apparently healthy individuals were assessed by ELISA, and in some high-risk groups, such as mentally ill hospitalized patients, a positive frequency of $12.1 \%(82 / 675)$ was observed ${ }^{14}$. A serological prevalence of $11.2 \%(137 / 1225)$ can be indirectly inferred for the Bolivian population through information related to the Bolivian immigrants in European countries, in which positivity rates of $9.7 \%$ and $13.4 \%$ were reported in 739 and 486 Bolivian migrants in Italy and Switzerland, respectively ${ }^{15}$.

The particularly high seroprevalence for strongyloidiasis observed in this study when compared to other studies employing a similar diagnostic approach must be further investigated. One of the greatest challenges related to the use of serological tests for epidemiological studies is the possibility of cross-reactivity with other helminthic infections. Therefore, the limitations of this study include the lack of data related to other intestinal parasites within the study area, in addition to the relatively small sample size and no access to other clinical and laboratory data on patients. Furthermore, certain clinical conditions which are more common among patients attending the laboratory might have created a bias towards a higher prevalence.

To our knowledge, this is the first study on the occurence of strongyloidiasis in the southern region of Minas Gerais. We provide serological evidence that strongyloidiasis is present in Alfenas, although it is important to confirm these findings in a larger and more representative population sample. The epidemiological data used in this study was limited to those currently residing in the city, and several aspects of the study require further investigation. Future epidemiological studies should identify individual risk factors so that appropriate interventions can be implemented.

\section{Ethical aspects}

Serum samples were provided by the LACEN without patient names to preserve their anonymity. Instead only their registration code, gender, age and place of residence were provided. The project was approved by the Research Ethics Committee of the Federal University of Alfenas (approval number 968,626; 27/02/2015).

\section{Acknowledgments}

To the team of the Central Laboratory of Clinical Analyses / Federal University of Alfenas (UNIFAL-MG).

\section{Conflict of interests}

The authors declare that there is no conflict of interest.

\section{REFERENCES}

1. World Health Organization (WHO). Strongyloidiasis. WHO; 2017 a. Available at: http://www.who.int/intestinal_worms/epidemiology/ strongyloidiasis/en. Accessed at: 28/11/2017.

2. Schär F, Trostdorf U, Giardina F, Khieu V, Muth S, Marti H, et al. Strongyloides stercoralis: global distribution and risk factors. PLoS Negl Trop Dis. 2013;7(7):e2288. 
3. Boddu S, Li Y, Zhou X, Wang C, Li J, et al. Strongyloidiasis - an insight into its global prevalence and management. PLoS Negl Trop Dis. 2014;8(8):e3018.

4. Levenhagen MA, Costa-Cruz JM. Update on immunologic and molecular diagnosis of human strongyloidiasis. Acta Trop. 2014;135:33-43.

5. Requena-Mendez A, Buonfrate D, Bisoffi Z, Gutiérrez JM. Advances in the diagnosis of human strongyloidiasis. Curr Trop Med Rep. 2014;1(4):207-15.

6. Instituto Brasileiro de Geografia e Estatística (IBGE). Cidades. Minas Gerais. Alfenas. IBGE 2017 Available at: https://cidades. ibge.gov.br/brasil/mg/alfenas/panorama . Accessed at 12/12/2017.

7. de Carvalho CJ, Levenhagen MA, Costa-Cruz JM. The detergent fraction is effective in the detection of $\operatorname{IgG}$ anti-Strongyloides stercoralis in serum samples from immunocompromised individuals. Parasitol. 2014;63(6):790-3.

8. World Health Organization (WHO). Soil-transmitted helminth infections. WHO;2017b .WHO Fact Sheet $N^{\circ}$ 366. Geneva. Updated September 2017.

9. Sudré AP, Macedo HW, Peralta RHS, Peralta JM. Diagnóstico da estrongiloidíase humana: importância e técnicas. Rev Patol Trop. 2006;35(3):173-84.
10. Paula FM, Costa-Cruz JM. Epidemiological aspects of strongyloidiasis in Brazil. Parasitology. 2011;138(11):1331-40.

11. Machado ER, Teixeira EM, Paula FM, Gonçalves-Pires MRF, Ueta MT, Costa-Cruz JM. Immunoparasitological diagnosis of Strongyloides stercoralis in garbage collectors in Uberlândia, MG, Brazil. Parasitol Latinoam. 2007;62(3-4):180-2.

12. Mota-Ferreira DML, Gonçalves-Pires MRF, Ferreira $\mathrm{Jr}$ A, Sopelete MC, Adballah VOS, Costa-Cruz JM. Specific IgA and IgG antibodies in paired serum and breast milk samples in human strongyloidiasis. Acta Trop. 2009;109(2):103-7.

13. Yori PP, Kosek M, Gilman RH, Cordova J, Bern C, Chavez CB et al. Seroepidemiology of strongyloidiasis in the Peruvian Amazon. Am J Trop Med Hyg. 2006;74(1):97-102.

14. Mercado R, Jercic MI, Alcayaga S, de Paula FM, Ueta MT, CostaCruz JM. Seroepidemiological aspects of human Strongyloides stercoralis infections in Chile. Rev Inst Med Trop Sao Paulo. 2007;49(4):247-9.

15. Spinicci M, Macchioni F, Mantella A, Gabrielli S, Roselli M, Mayaregua DR et al. Seroepidemiological trend of strongyloidiasis in the Bolivian Chaco (1987-2013) in the absence of disease-specific control measures. Trop Med Int Health. 2017;22(11):1457-62. 